\title{
Stochastic effects in intercellular calcium spiking in hepatocytes
}

\author{
M. E. Gracheva ${ }^{1}$, R. Toral ${ }^{2}$, J. D. Gunton ${ }^{1}$ \\ ${ }^{1}$ Department of Physics, Lehigh University, \\ Bethlehem, PA 18015 \\ 2 Instituto Mediterraneo de Estudios Avanzados, \\ CSIC-UIB, E-07071 Palma de Mallorca, Spain
}

\begin{abstract}
We carry out a Monte Carlo simulation of stochastic effects for two models of intercellular calcium wave propagation in rat hepatocytes. Both models involve gap junction diffusion by a second messenger. We find that in general the stochastic effects improve agreement with experiment, for a reasonable choice of model parameters. Both stochastic models exhibit baseline fluctuations and variations in the peak heights of $\mathrm{Ca}^{2+}$. In addition, we find for one model that there is a distribution of latency times, rather than a single latency time, with a width which is comparable to the experimental observation of spike widths. We also find for the other model with low gap junction diffusion that it is possible for cell multiplets to oscillate independently initially, but to subsequently become synchronized.
\end{abstract}

Keywords: Stochastic, calcium waves, calcium signaling, gap junctions, hepatocytes.

\section{INTRODUCTION}

Cell to cell signals control the development of multicellular organisms as well as most of their functions (Goldbeter, 1996). These signals have many different manifestations and provide excellent examples of nanoscale biology. Calcium signaling plays a particularly important role in cell communication. Single hepatocytes respond to hormonal stimulation with repetitive spikes in intracellular $\mathrm{Ca}^{2+}$ concentration (Thomas et al., 1996; Thomas et al., 1991; Thomas et al., 1995). Multiplets of hepatocytes can exhibit well-coordinated spiking, known as intercellular $\mathrm{Ca}^{2+}$ waves. Such intercellular communication can take different forms, including gap junction coupling, paracrine signaling and the recently discovered extracellular calcium signaling (Höfer et al., 2000). In particular, diffusion of second messengers through gap junctions appears to be responsible for intercellular calcium waves in tracheal ciliated cells (Sneyd et al., 1995; Sanderson et al., 1990), glial cells (Charles et al., 1992), pancreatic acinar cells (Loessberg-Stauffer et al., 1993; Yule et al., 1996) and other types (Sanderson et al., 1994).

There exist two different types of experimental studies of such waves. In one class, a single cell of a cultured monolayer is stimulated mechanically, inducing the propagation of $\mathrm{Ca}^{2+}$ waves in the adjacent cells. Such studies have been carried out on tracheal epithelial cells (Hansen et al., 1993) and endothelial cells. Sneyd et al. (Sneyd et al., 1995; Sneyd et al., 1998) have proposed a model for these intercellular waves, which assumes gap junctional diffusion of $\mathrm{IP}_{3}$ between adjacent cells. Mechanical stimulation of a single cell produces $\mathrm{IP}_{3}$ within the cell, which in turn causes the release of $\mathrm{Ca}^{2+}$ from internal stores in the form of an intracellular $\mathrm{Ca}^{2+}$ wave. Diffusion of $\mathrm{IP}_{3}$ between cells then initiates calcium waves in adjacent cells. This process continues as long as the amount of $\mathrm{IP}_{3}$ entering a given cell is sufficient to induce a $\mathrm{Ca}^{2+}$ wave. In another class of experiments, studies are carried out on freshly isolated systems of connected cells that are globally stimulated by hormones (Loessberg-Stauffer et al., 1993; Nathanson et al., 1992; Combettes et al., 1994; Nathanson et al., 1995; Robb-Gaspers et al., 1995). An interesting feature of these studies for liver cells (which are tightly coupled by gap junctions) is the sequential pattern of $\mathrm{Ca}^{2+}$ spiking in the different connected cells, which creates the appearance of $\mathrm{Ca}^{2+}$ waves (Nicholson et al., 1987).

Some recent papers have studied the mechanisms that control the coordination and intercellular propagation of calcium waves induced in rat hepatocytes (studying propagation of such intercellular $\mathrm{Ca}^{2+}$ waves in doublet and triplet cells). A first paper by Tordjmann et al. (Tordjmann et al., 1997) studied calcium waves induced by noradrenaline and showed that gap junction coupling is necessary for the coordination of the oscillations between the different cells. The authors also demonstrated that it is necessary to have hormone stimulation at each hepatocyte in order to have cell-cell calcium signal propagation. Furthermore, they also found that there were functional differences between adjacent hepatocytes. A subsequent paper by the same authors (Tordjmann et al., 1998) continued these studies, combining single-cell studies with experiments on cell populations isolated from the peripheral (periportal) and central (perivenous) zones of the liver cell plate. They found strong evidence that the sequential pattern of calcium responses to vasopressin in these multicellular rat hepatocyte systems was due to a gradient of cell sensitivity (from cell to cell) for the hormone. The first cell to respond had the greatest sensitivity to the global stimulus, while the last cell to respond had the least sensitivity. This is an important result, since such gradients may impose an orientation on calcium waves in liver cells and provide a pacemaker-like mechanism for regulating intercellular communication in the liver. Based upon these experimental studies, two models have been put forward in order to explain the observed results.

The first model is due to Dupont et al. who in (Dupont et al., 2000) studied a model based on junctional coupling of multiple hepatocytes which differ in their sensitivity to the hormonal stimulus. As a consequence of this difference, the intrinsic frequency 
of intracellular calcium oscillations also varies from cell to cell. These oscillators are coupled by an intercellular messenger, which could be either $\mathrm{Ca}^{2+}$ or inositol 1,4,5-triphosphate $\mathrm{IP}_{3}$. The model yielded intercellular waves that were confirmed experimentally (Dupont et al., 2000). The authors also presented experimental evidence that the degree of synchronization is greater for the first few spikes, in agreement with the prediction of their model. They also presented evidence that suggested, within the context of their model, that IP3 diffusion through gap junctions (rather than $\mathrm{Ca}^{2+}$ diffusion) plays the dominant role in the synchronization of intercellular spiking.

An alternative model has also been proposed by Höfer (Höfer et al., 1999) to explain the experimental results obtained in the first paper by Tordjmann et al (Tordjmann et al., 1997). Höfer noted that this experiment revealed a rather large variability in oscillator frequency between adjacent cells, which he argued is likely to be of random nature. As a consequence he studied the possibility that this originates from random variations in the structural properties of cells (cell size, cell shape, or ER content). In addition, $\mathrm{Ca}^{2+}$ was assumed to be the second messenger (Höfer et al., 1999). His results were in reasonable agreement with those of Tordjmann et al. (Tordjmann et al., 1997).

Although we are not in a position to judge the relative merits of the two models, both are relatively successful and quite interesting. However, they both have limitations. For example, the calcium spikes in the Dupont et al. are extremely sharp, whereas the experimental spikes have a finite width. Höfer's model predicts more reasonable spike widths, but predicts an intercellular synchronization at low stimulus that seems inconsistent with experiment (cf. Section 2). In addition, both models are deterministic, described by differential equations with boundary conditions for the cell multiplets and with diffusion between cells. Such models, however, do not produce stochastic effects such as fluctuations in the baseline values of calcium and variations in the amplitudes and widths of the spikes that have been seen experimentally (Tordjmann et al., 1997; Tordjmann et al., 1998). Indeed, since the number densities of intracellular signaling molecules are typically low (of order 1 to $10^{2} \mu m^{-3}$, one would expect stochastic effects to be important (Stundzia et al., 1996; Kraus et al., 1996). To obtain a better explanation of the experimental results, we have therefore studied stochastic versions of the above two models. Our simulation is based on a Monte Carlo method due to Gillespie (Gillespie, 1976; Gillespie, 1977). Stochastic models of intracellular Ca ${ }^{2+}$ spiking for a variety of cell types have been studied previously (Kraus et al., 1996; Prank et al., 1998; Keizer and Smith, 1998; Keizer et al., 1998; Falcke et al., 2000).

The outline of our paper is as follows. In Section II we define and study a stochastic version of Höfer's model. In Section III we study a stochastic version of Dupont et al.'s model. In both sections we compare our results with those of experiment. Finally in Section IV we present a brief conclusion.

\section{CALCIUM SYNCHRONIZATION OF HETEROGENEOUS CELLS}

We first study a stochastic version of the deterministic model proposed by Höfer (Höfer et al., 1999) to explain the synchronization of calcium oscillations in heterogeneous hepatocyte cells found by Tordjmann et al. (Tordjmann et al., 1998). His model of intracellular dynamics is similar to earlier models (Somogyi et al., 1991; Dupont and Goldbeter, 1993), but includes calcium inhibition of receptors (Bezprozvanny and Ehrlich, 1995; DeYoung et al., 1991). As noted above, he assumed that the rather large variability in intrinsic oscillator frequencies observed by Tordjmann et al. is due to random heterogeneities of structural properties (such as cell size, cell shape and ER content). He also argued that since there seems to be no feedback on PLC in hepatocytes (Bird et al., 1997) and since non-metabolizable analogies of $\mathrm{IP}_{3}$ can also produce oscillations (Thomas et al., 1991) there is no involvement of $\mathrm{IP}_{3}$ in the mechanism of oscillations. He thus assumed that the concentration of IP 3 rapidly reaches a steady-state value (which can differ for different cells) that is treated as a parameter of the model. In addition, he argued that since calcium oscillations may cause continuously changing junctional fluxes of calcium, the intercellular synchronization might be due to a $\mathrm{Ca}^{2+}$ flux across cellular gap junctions. Höfer's model considers a series of $j=1,2, \ldots, N$ connected cells. We will be considering in this study single cells, $N=1$, doublets, $N=2$ and triplets, $N=3$. Let $x_{j}$ and $z_{j}$ be, respectively, the cytosolic calcium concentration and the free calcium content in cell $j$. The latter is defined as $z_{j}=x_{j}+\left(C_{E R} / C_{C}\right) y_{j}$, where $y_{j}$ denotes the free calcium concentration in the ER and $C_{C}=V_{C}\left(1+B_{o} / K_{B}\right)$ and $C_{E R}=V_{E R}\left(1+B_{o} / K_{B}\right)$ are effective volumes of the cytosol and endoplasmic reticulum, respectively. $V_{C}$ and $V_{E R}$ are the real volumes of the cytosol and ER, and where $K_{B}$ and $B_{o}$ are a dissociation constant and the total concentration of calcium binding sites respectively.

After some simplification Höfer obtained the following deterministic model for the time evolution of the $x_{j}$ and $z_{j}$ variables in the case of a doublet, $N=2$ :

$$
\begin{aligned}
\frac{d x_{j}}{d t} & =\rho_{j}\left(\nu_{0}+\nu_{c} \frac{P_{j}}{K_{0}+P_{j}}-\nu_{4} \frac{x_{j}^{2}}{K_{4}^{2}+x_{j}^{2}}+\frac{\alpha k_{r}\left(x_{j}, P_{j}\right)}{\beta_{j}}\left(z_{j}-\left(1+\beta_{j}\right) x_{j}\right)\right. \\
& \left.-\alpha \nu_{3} \frac{x_{j}^{2}}{K_{3}^{2}+x_{j}^{2}}\right)+\gamma\left(x_{i}-x_{j}\right)
\end{aligned}
$$




$$
\frac{d z_{j}}{d t}=\rho_{j}\left(\nu_{0}+\nu_{c} \frac{P_{j}}{K_{0}+P_{j}}-\nu_{4} \frac{x_{j}^{2}}{K_{4}^{2}+x_{j}^{2}}\right)+\gamma\left(x_{i}-x_{j}\right)
$$

The last term, proportional to $\gamma$, denotes diffusion between cells. The index pairs $(i, j)=(1,2)$ and $(2,1)$. The system can be easily generated to the case of more than two cells. In these equations $P_{j}$ is the $\mathrm{IP}_{3}$ concentration in cell $j$. The $\mathrm{IP}_{3} \mathrm{R}$ release function $k_{r}\left(x_{j}, P_{j}\right)$ describes the gating kinetics of the $\mathrm{IP}_{3}$ receptor $\mathrm{R}$ and it is given by

$$
k_{r}\left(x_{j}, P_{j}\right)=k_{1}\left[\frac{d_{2}\left(d_{1}+P_{j}\right) P_{j} x_{j}}{\left(d_{p}+P_{j}\right)\left(d_{a}+x_{j}\right)\left(d_{2}\left(d_{1}+P_{j}\right)+x_{j}\left(d_{3}+P_{j}\right)\right)}\right]^{3}+k_{2}
$$

an expression based on earlier theoretical work (DeYoung et al., 1991; Li et al., 1994). The parameters $\rho=A_{P M} / C_{C}$, $\alpha=A_{E R} / A_{P M}$ and $\beta=C_{E R} / C_{C}$ define various structural characteristics of the cell and account for the heterogeneous behavior of different cells. The variables $A_{E R}$ and $A_{P M}$ are the areas of the ER and plasma membrane respectively. $C_{C}$ and $C_{E R}$ are effective cytosolic and ER volumes defined as $C_{C}=V_{C}\left(1+B_{o} / K_{B}\right)$ and $C_{E R}=V_{E R}\left(1+B_{o} / K_{B}\right)$ respectively. $K_{B}$ is a dissociation constant and $B_{o}$ is the total concentration of calcium binding sites. Höfer estimates that this cytosolic calcium buffering factor $\left(1+B_{o} / K_{B}\right)$ ranges from 20 to 100 or so depending on cell type and chooses it as 75 in his calculation. The definitions of the other parameters as well as their values are given in (Höfer et al., 1999). Table I summarizes the values we adopt for these parameters in the present paper.

The above set of Höfer's equations is deterministic and completely ignores the fluctuations that appear from the fact that the chemical reactions do not occur uniformly and continuously in time. Gillespie's method considers specifically that (a) the concentration of molecular species can only vary by a discrete amount and (b) the chemical reaction itself is a stochastic process that occurs with a certain rate. Therefore, it is not possible to determine which reaction will occur next, but rather which is the probability that a given reaction will take place.

In accordance with Gillespie's method, we introduce the number populations of cell $j$ as $X_{j}$ and $Z_{j}$, such that the concentrations of the reactants are obtained as:

$$
x_{j}=\frac{X_{j}}{\Omega} ; \quad z_{j}=\frac{Z_{j}}{\Omega}
$$

In this model $\Omega$ is the volume of the cytosolic compartment of the cell, with fluctuation effects being most notable for small $\Omega$. The population numbers $X_{j}$ and $Z_{j}$ can vary by discrete, integer, amounts according to some probability that reflects the possible reactions taking place in the system. The possible events and their reaction constants are defined in table II. Consistent with the previous system of differential equations we choose the following expressions for the rates:

$$
\begin{aligned}
a_{j}^{(1)} & =\Omega \rho_{j}\left(\nu_{0}+\nu_{c} \frac{P_{j}}{K_{0}+P_{j}}+\frac{\alpha k_{r}\left(x_{j}, P_{j}\right)}{\beta_{j}} z_{j}\right), \\
a_{j}^{(2)} & =\Omega \rho_{j}\left(\nu_{4} \frac{x_{j}^{2}}{K_{4}^{2}+x_{j}^{2}}+\frac{\alpha k_{r}\left(x_{j}, P_{j}\right)}{\beta_{j}}\left(1+\beta_{j} x_{j}\right)+\alpha \nu_{3} \frac{x_{j}^{2}}{K_{3}^{2}+x_{j}^{2}}\right), \\
a_{j}^{(3)} & =\Omega \rho_{j}\left(\nu_{0}+\nu_{c} \frac{P_{j}}{K_{0}+P_{j}}\right) \\
a_{j}^{(4)} & =\Omega \rho_{j} \nu_{4} \frac{x_{j}^{2}}{K_{4}^{2}+x_{j}^{2}}, \\
a_{j}^{(5)} & =\gamma \max \left(X_{j+1}-X_{j}, 0\right), \\
a_{j}^{(6)} & =\gamma \max \left(X_{j}-X_{j+1}, 0\right), \\
a_{j}^{(7)} & =\gamma \max \left(X_{j+1}-X_{j}, 0\right), \\
a_{j}^{(8)} & =\gamma \max \left(X_{j}-X_{j+1}, 0\right),
\end{aligned}
$$

and the convention that, whenever they appear, we define $X_{0}=Z_{0}=X_{N+1}=Z_{N+1}=0$ as the boundary condition for diffusion. The stochastic simulation proceeds (Gillespie, 1977) by choosing randomly one of the $8 \times N$ events with a probability proportional to the reaction rate. Once the event is selected, the number populations change accordingly and time increases by a given amount.

Following (Höfer et al., 1999) we consider a spherical cell with a radius of $6 \mu \mathrm{m}$, with a cell volume of about $900 \mu \mathrm{m}^{3}$, one third of which is a cytosolic volume of about $300 \mu \mathrm{m}^{3}$. We have considered $\Omega$ to be essentially a parameter controlling the size of the fluctuations. We display our results for $\Omega=300 \mu \mathrm{m}^{3}$, i.e. the cytosolic cell volume. Fig. 1 shows the calcium oscillations 
for one isolated cell in our stochastic model for $\Omega=300$ and $10^{5}$ respectively. Note that the result for large $\Omega=10^{5}$ agrees with the deterministic limit (Höfer et al., 1999).

Since the cell-cell permeability $\gamma$ that enters in the gap junction coupling is not known, we study the calcium oscillations for a range of permeability values. To determine the maximum value of $\gamma$ we should use in the stochastic model we simulated the experimental study of the doublet of hepatocytes, namely, first with only one of the cells stimulated with a hormonal input (local perfusion) and then with both cells simultaneously stimulated (global perfusion). From the experimental results we know that local perfusion is not sufficient for coordinated oscillations. Global perfusion of both cells, on the other hand, produces a well synchronized $\mathrm{Ca}^{2+}$ oscillation in the two cells. In Fig. 2 (a,b,c) we show our results of stimulating only one cell in the doublet. We increase the cell - cell permeability to find the largest value that will not produce $\mathrm{Ca}^{2+}$ spiking in the second cell. We see that the two cells respond differently, with different periods of oscillations; in neither case does the unstimulated cell show $\mathrm{Ca}^{2+}$ oscillations. But if we stimulate both hepatocytes they respond with well coordinated $\mathrm{Ca}^{2+}$ oscillations. This yields the value of $\gamma_{\max }=0.07 \mathrm{~s}^{-1}$.

Next we study the behavior of two connected hepatocytes. To simulate the experimental situation of two slightly different cells, we choose different structural parameters, with $\beta_{1}=0.15, \beta_{2}=0.2$. We do not follow Höfer in our choice of structural parameters since for his choice of parameters $\left(\beta_{1}=0.2, \beta_{2}=0.2\right)$ for a given volume $\Omega=300$ we could not obtain a well synchronized response from two coupled cells. We also choose slightly different values of $\rho$ (than Höfer) to have better synchronized oscillation patterns. The results of the simulation are shown in Figs. 3(a,b,c) for $\gamma=0.0 s^{-1}, 0.07 \mathrm{~s}^{-1}$. The calcium oscillations in the two cells are totally uncoordinated if the membrane permeability is set to zero, as should be the case (Fig. 3a). For a value of the permeability $\gamma=0.07 \mathrm{~s}^{-1}$ we find 1:1 locking (Fig. 3b). Fig. 3c shows the result for $\gamma=0.07 \mathrm{~s}^{-1}$ in the deterministic limit of large $\Omega$, which is in agreement with Höfer's results for this choice of parameters.

We have also simulated the experimental situation in which, after a few coordinated oscillations (between 200-500s), the membrane permeability between cells is blocked in such a way as to prevent $\mathrm{Ca}^{2+}$ from passing through the membrane $(\gamma$ is set to zero in the model). In this case the cells lose synchronization, but after washing the chemical responsible for the blocking, the cells regain synchronization. This behavior is clearly seen in Fig.4a. The stochastic model yields a variation in the amplitude of oscillations and fluctuations in the baseline value of $\mathrm{Ca}^{2+}$, in agreement with the experimental results. These effects are absent in the deterministic limit of the model, shown in Fig.4b.

Finally, we model the experimental study of a triplet of hepatocytes, in which one can see synchronized intercellular signaling. However, if a heparin treatment is applied to the intermediate cell the synchronized spiking between the first and third cells is destroyed. In addition, the calcium oscillations of the middle cell are altered. Fig.5a show the results of our simulation. It can be seen that after the heparin application at $t=200 \mathrm{~s}$ there are no calcium oscillations in the second cell, and the first and third cells in the triplet spike asynchronously. These results are in good agreement with the experimental results (Tordjmann et al., 1998). We also show the results of going to the deterministic limit of large cell volume in Fig.5b, which are in agreement with the original study, as to be expected.

The result of simulation of a cell triplet with the membrane permeability between cells set to zero is presented in Fig.6. It can be clearly seen that cells that are not connected by gap junctions exhibit uncoordinated calcium signaling. Experiments also show the absence of coordination among the calcium signals in connected hepatocytes at low concentrations of stimuli. The cells respond in an asynchronous fashion because the relative differences in the levels of $\mathrm{IP}_{3}$ are important. To simulate this situation we have conducted the following numerical experiment. First we applied a low stimulation level $P=1 \mu \mathrm{M}$ to all three cells in the triplet, taking into account that cells can vary in structural properties (with $\rho_{1}=0.025, \rho_{2}=0.015$, and $\rho_{3}=0.02$ ). Note that calcium oscillations become synchronized with time (Fig. 7a). This continues to be the case even for membrane permeability constants as small as $\gamma=0.03$ (result not shown). This behavior has not been seen experimentally. Next, we introduce a gradient in the $\mathrm{IP}_{3}$ concentration, with $P_{1}=1.2, P_{2}=1.1$ and $P_{3}=1.0$, for three structurally identical cells with $\rho_{1}=\rho_{2}=\rho_{3}=0.02$. Fig. 7b shows that calcium oscillations that are initially synchronous become asynchronous with time due to noise, and then again become synchronous. Although this effect has not been seen experimentally, it would be very interesting to have experimental observations of calcium oscillations over long time intervals for medium stimulation levels, since it is possible that even cells that are initially unsynchronized may become synchronized later on.

\section{IP S $_{3}$ SYNCHRONIZATION VIA HORMONAL SENSITIVITY GRADIENT}

The second model we study is due to Dupont et al. (Dupont et al., 2000) and considers IP 3 as the second messenger responsible for coordination of $\mathrm{Ca}^{2+}$ signaling in connected hepatocytes. This model is based on the experimental observation that the number of external receptors on a hepatocyte membrane depends on its location in the liver cell plate (Tordjmann et al., 1998). Thus the authors consider a model of a multiplet of gap junction connected cells, with a small variation in the individual cell frequencies. The dynamics of each cell $j$ is described by a set of three dynamical variables $R_{j}^{\text {des }}, x_{j}$ and $y_{j}$. These are the fraction of inactive $\mathrm{IP}_{3}$ receptors, the concentration of cytosolic $\mathrm{Ca}^{2+}$ and the concentration of $\mathrm{IP}_{3}$, respectively. The equations of motion are taken to be 


$$
\begin{aligned}
\frac{d R_{j}^{\text {des }}}{d t} & =k_{+} x_{j}^{4} \frac{1-R_{j}^{\text {des }}}{1+\left(x_{j} / K_{a c t}\right)^{3}}-k_{-} R_{d e s} \\
\frac{d x_{j}}{d t} & =k_{1}\left(b+I R_{a}\right)\left[C a_{t o t}-x_{j}(\alpha+1)\right]-V_{M P} \frac{x_{j}^{2}}{x_{j}^{2}+K_{P}^{2}} \\
\frac{d y_{j}}{d t} & =V_{j}^{P L C}-V_{K} \frac{y_{j} x_{j}^{2}}{\left(K_{K}+y_{j}\right)\left(x_{j}^{2}+K_{d}^{2}\right)}-V_{P H} \frac{y_{j}}{K_{P H}+y_{j}}
\end{aligned}
$$

where

$$
I R_{a}=\frac{1-R_{j}^{\text {des }}}{1+\left(K_{a c t} / x_{j}\right)^{3}} \frac{y_{j}^{3}}{K_{I P}^{3}+y_{j}^{3}} .
$$

Besides these evolution equations, there is also diffusion of calcium inside each cell as well as intercellular diffusion of $\mathrm{IP}_{3}$, with the latter providing the coupling between adjacent cells. The $\mathrm{IP}_{3}$ diffusion is modelled by assuming that at each boundary between two cells:

$$
D_{I P} \frac{\partial y^{-}}{\partial s}=D_{I P} \frac{\partial y^{+}}{\partial s}=F_{I P}\left(y^{+}-y^{-}\right) .
$$

where the superscripts + and - indicate the $\mathrm{IP}_{3}$ concentration at the right and left limits of the border, respectively. The spatial coordinate is indicated by $s$. We consider one-dimensional cells $20 \mu \mathrm{m}$ long, each containing 20 grid points.

We study, using Gillespie's method, a stochastic version of this model for different cell volumes and for a range of values of the cell-cell permeability. We consider a cell of $20 \mu m \times 20 \mu m \times 1 \mu m$ in size (which gives us $\Omega=400$ for our stochastic simulations), as assumed by Dupont et al. Fig. 8 presents the results of our simulation for a single cell for some values of $\Omega$, with the deterministic limit corresponding to large $\Omega=50000$. The results of our stochastic simulation in this deterministic limit are consistent with (Dupont et al., 2000), as to be expected. In contrast to the deterministic model where the induction time (latency of cell) depends only on the stimulus strength, we find a distribution of induction times in the stochastic model, due to fluctuations in the calcium concentration. Fig.9 shows the distribution of induction times for one stimulated cell with $V^{P L C}=2 \times 10^{-3} \mu \mathrm{M} / \mathrm{s}$. As there does not appear to be any systematic experimental study of such a distribution, we have no data to compare our results with. It is also the case that the calcium spikes in these experiments have a width of $20-30 s$, which means that would be difficult to see fluctuations in the central position of the spikes.

For two connected cells we determine the cell-cell permeability following reference (Dupont et al., 2000), such that a doublet of cells, with only one cell doped with stimulant, exhibits calcium oscillations only in the stimulated cell (as has been shown experimentally). Fig. 10a presents these data. The results obtained from this stochastic model are in agreement with experiment (Dupont et al., 2000), although the cell to cell permeability $F_{I P}=0.35 \mu \mathrm{m} / \mathrm{s} \mathrm{differs} \mathrm{somewhat} \mathrm{from} \mathrm{that} \mathrm{in} \mathrm{the} \mathrm{deterministic}$ model. We have to use a smaller value for the permeability because noise in the baseline produce spikes in the second, nonstimulated cell if the permeability is larger then $0.35 \mu \mathrm{m} / \mathrm{s}$. We use $V_{1}^{P L C}=2.5 \times 10^{-3} \mu \mathrm{M} / \mathrm{s}, V_{2}^{P L C}=6.5 \times 10^{-4} \mu \mathrm{M} / \mathrm{s}$. We decreased the value of $V^{P L C}$ to obtain agreement with the experimental values of the average induction time. We also find that the stochastic model with the parameters of reference (Dupont et al., 2000) reproduces the deterministic model in the limit of large cell volume. This is to be expected, as in that limit fluctuation effects become negligible. Fig. 10b shows the results of the stochastic model in the deterministic limit for the same parameters as in (Dupont et al., 2000) (permeability $F_{I P}=0.88 \mu \mathrm{m} / \mathrm{s}$, $V_{1}^{P L C}=2.77 \times 10^{-3} \mu \mathrm{M} / \mathrm{s}, V_{2}^{P L C}=6.5 \times 10^{-4} \mu \mathrm{M} / \mathrm{s}$ ). Another distinguishing feature from the deterministic model is that stochastic effects produce a variation in the spike amplitudes, as was clearly seen in Fig. 8.

Figure 11a shows the result of the simulations for two connected, both stimulated, cells. These cells don't go out of phase as rapidly as in the deterministic model. Figure $11 \mathrm{~b}$ shows two cells with $\mathrm{IP}_{3}$ diffusion suppressed $\left(F_{I P}=0.0 \mu \mathrm{m} / \mathrm{s}\right)$. Figure $11 \mathrm{c}$ shows our results in the deterministic limit for large volume, for the same parameters as in (Dupont et al., 2000).

The experimental results exhibit more synchronization between cells than in this stochastic model. However, the stochastic model yields better agreement with experiment in terms of the variation in amplitudes and period variations (for $\Omega=400$ ). As noted in the introduction, the narrow width of the calcium peaks is a weakness of this model. Also note that in this model the cell with smaller sensitivity can spike first depending on random fluctuations in the second cell (at $\Omega=400$ ).

For three connected cells we find in general the same results as for two connected cells, namely the cells spike in phase for several times, but then lose synchronization, as can be seen in Fig.12(a,b). Finally, we simulate the effect of washing just before the third cell spikes. After restoration of the agonist, the third cell normally spikes first (Fig. 13), but this is not always the case and depends on fluctuations. 


\section{CONCLUSION}

We have studied calcium oscillations in connected hepatocytes for two different stochastic models of calcium dynamics. The first model (Höfer et al., 1999) describes calcium dynamics between the endoplasmic reticulum and cytosol, with diffusion of calcium between connected cells. In the second model (Dupont et al., 2000) connected cells have a gradient in IP 3 sensitivity with diffusion of $\mathrm{IP}_{3}$ between neighboring cells. Both models are described by a system of nonlinear differential equations. We have solved these two models using a Monte Carlo approach, considering each term in a model as a specific reaction occurring with a certain reaction rate. Our stochastic models are in better agreement with experiment than are the deterministic models. Both stochastic models exhibit baseline fluctuations and variations in peak heights. The baseline fluctuations are somewhat smaller in the second model with diffusion of $\mathrm{IP}_{3}$ between cells, due to an averaging of calcium concentration over the cell volume, as well as to model characteristics. The drawback of this model is the very narrow width of the calcium spikes. This results in a distribution of latency times with a width of about $15 \mathrm{~s}$ which is about the same order as the spike widths observed in experiments. This would make it somewhat difficult to see such a distribution. In addition, the model could be significantly improved by a modification that would yield a finite width for the spikes. When compared to the deterministic model, one finds that a smaller permeability coefficient is needed in the stochastic model, since calcium fluctuations on the baseline level give rise to calcium oscillations in the non-stimulated cell if only one cell is stimulated. It would be useful to have experimental results for calcium oscillations on a much longer time scale than is normally presented, since the stochastic model shows that doublets of cells can lose synchronization of calcium oscillations, but can subsequently regain it.

The model (Höfer et al., 1999) with diffusion of $\mathrm{Ca}^{2+}$ between cells reproduced the experimental results for two cells with only one of the cells stimulated. The model reproduces the experimental behavior in which the unstimulated cell does not show calcium oscillations. Also, in accordance with experiments, when the two cells are both globally stimulated, well coordinated calcium oscillations can be seen in both cells. In the stochastic model the frequency of final oscillations of coupled cells was slightly smaller than the frequency of the cell with larger frequency oscillations in the doublet (when the cells are not connected by gap junction diffusion). We have found that although this model works quite well for average stimuli strength, the model does not reproduce the observed experimental response of cells at low stimuli with different structural parameters for three connected cells. This is also true when the cells have different $\mathrm{IP}_{3}$ sensitivity. Instead of a gradual loss of synchronization, cells remain synchronized with a larger period of spiking. They also remain synchronized gap junction permeabilities as small as $\gamma=0.035 \mathrm{~s}^{-1}$. With a further decrease of gap junction permeability, the cells initially do not oscillate together, but can subsequently become synchronized. All the results of both deterministic models have been reproduced for their stochastic versions, in the limit of large volume, as should be the case. Finally we conclude that it is important to take into account stochastic effects in modeling calcium oscillations in connected hepatocytes.

Acknowledgments: This work was supported in part by NSF Grant number DMR9813409 and the DGES (Spain) projects PB97-0141-C02-01 and BFM2000-1108. We thank K. Prank for bringing this problem to our attention and for helpful discussions. We also wish to acknowledge helpful correspondence with G. Dupont. 


\section{REFERENCES}

Bezprozvanny, I. and B. E. Ehrlich. 1995. The inositol 1,4,5-triphosphate (InsP 3 ) receptor. J. Membr. Biol. 145:205-216.

Bird, G. S. J., J. F. Obie and J. W. Putney. 1997. Effect of cytoplasmic $\mathrm{Ca}^{2+}$ on $(1,4,5) \mathrm{IP}_{3}$ formation in vasopressi-activated hepatocytes. Cell Calcium. 21:253-256.

Charles, A. C., C. C. G. Naus, D. G. Zhu, Kidder, E. R. Dirksen and D. L. Sanderson. 1992. Intercellular calcium signaling via gap junctions in glioma cells. J. Cell. Biol. 118:195-201.

Combettes, L., D. Tran, T. Tordjmann, J. Laurent, B. Berthon and M. Claret. 1994. Sequential activation of hormone-mediated $\mathrm{Ca} 2+$ signals in multicellular systems of rat hepatocytes. Biochem. J. 304:585-594.

DeYoung, G. W. and J. Keizer. 1991. A single-pool inositol 1,4,5-triphosphate receptor-based model for agonist-stimulated oscillations in $\mathrm{Ca}^{2+}$ concentration. Proc. Natl. Acad. Sci. USA 89:9895-9899.

Dupont, G. and A. Goldbeter. 1993. A one-pool model for $\mathrm{Ca}^{2+}$ oscillations involving $\mathrm{Ca}^{2+}$ and inositol 1,4,5-triphosphate as co-agonists for $\mathrm{Ca}^{2+}$ release. Cell Calcium 22:311-322.

Dupont, G., Th. Tordjmann, C. Clair, St. Swillens, M. Claret and L. Combettes. 2000. Mechanism of receptor-oriented intercellular calcium wave propagation in hepatocytes. FASEB J. 14:279-289.

Falcke M., L. Tsimring and H. Levine. 2000. Stochastic spreading of intracellular $\mathrm{Ca}^{2+}$ release. Phys. Rev. E 62:2636-2643.

Gillespie, D.. 1976. A general method for numerically simulating the stochastic time evolution of coupled chemical reactions.

J. of Comp. Phys. 22:403-434.

Gillespie, D.. 1977. Exact sthochastic simulation of coupled chemical reactions. J. of Phys. Chem. 81:2340-2361.

Goldbeter A.. 1996. Biochemical Oscillations and Cellular Rhythms, Cambridge University Press.

Hansen, M., S. Boitano, E. R. Dirksen and M. J. Sanderson. 1993. Intercellular calcium signaling induced by extracellular adenosine 5'-triphosphate and mechanical stimulation in airway epithelial cells. J. Cell. Sci. 106:995-1004.

Hansen, M., S. Boitano, E. R. Dirksen, M. J. Sanderson. 1995. A role for phospholipase C activity but not ryanodine receptors in the initiation and propagation of intercellular calcium waves. J. Cell Sci. 106:2583-2590 .

Höfer, A. M., S. Curci, M. A. Doble, E. M. Brown, E. M. and D. I. Soybel. 2000. Intercellular communication mediated by the extracellular calcium-sensing receptor. Nature Cell Biol. 2:392-398.

Höfer, Th.. 1999. Model of intercellular calcium oscillations in hepatocytes: synchronization of hetegogeneous cells. Biophys. J. 77:1244-1256.

Keizer, J. and G. D. Smith. 1998. Spark-to wave transition: salitatory transmission of calcium waves in cardiac myocytes. Biophys. Chem. 72:87-100.

Keizer, J., G. D. Smith, S. Ponce-Dawson and J. E. Pearson. 1998. Saltatory propagation of $\mathrm{Ca}^{2+}$ waves by $\mathrm{Ca}^{2+}$ sparks. Biophys. J. 75:595-600.

Kraus, M., B. Wolf and B. Wolf. 1996. Crosstalk between cellular morphology and calcium oscillation patterns. Cell Calcium 19:461-472.

$\mathrm{Li}, \mathrm{Y}$. X. and J. Rinzel. 1994. Equations for $\mathrm{InsP}_{3}$ receptor-mediated $[\mathrm{Ca}]_{i}$ oscillations derived from a detailed kinetic model: Hodgkin-Huxley-like formalism. J. Theor. Biol. 166:461-473.

Loessberg-Stauffer, P., H. Zhao, K. Luby-Phelps, R. L. Moss, R. A. Star and S. Muallem. 1993. Gap-junction communication modulates $\left(\mathrm{Ca}^{2+}\right)_{i}$ oscillations and enzyme secretion in pancreatic acini. J. Biol. Chem. 268:19769-19775.

Nathanson, M., A. Burgstahler, A. Mennone, M. Fallon, C. Gonzalez and J. Saez. 1995. Ca ${ }^{2+}$ waves are organized among hepatocytes in the intact organ. Am. J. Physiol. 32:G167-G171.

Nathanson, M., and A. Burgstahler. 1995. Coordination of hormone-induced calcium signals in isolated rat hepatocyte couplets. Demonstration with confocal microscopy. Mol. Biol. Cell 3:113-121.

Nicholson, B., R. Dermietzel, D. Teplow, O. Traub, K. Willecke and J. P. Revel. 1987. Two homologous protein components of hepatic gap junctions. Nature 329:732-734.

Prank, K., U. Ahlvers, F. Baumgarte, H.G. Musmann, A. von zur Muehlen, C. Schoefl, G. Brabant. 1998. Stochastic model of intracellular calcium spikes. Meditinische Biometrie, 385-388.

Robb-Gaspers, L. and A. Thomas. 1995. Coordination of $\mathrm{Ca}^{2+}$ signaling by intercellular propagation of $\mathrm{Ca}^{2+}$ waves in the intact liver. J. Biol. Chem. 270:8102-8107.

Sanderson, M. J., A. C. Charles and E. Dirksen. 1990. Mechanical simulation and intercellular communication increases intracellular $\mathrm{Ca}^{2+}$ in epithelial cells. Cell. Regulat. 1:585-596.

Sanderson, M. J., A. C. Charles, S. Boitano and E. R. Dirksen. 1994. Mechanisms and function of intercellular calcium signaling. Mol. Cell. Endocrinol. 98:172-187.

Sneyd, J., B. Wetton, A. Charles and M. Sanderson. 1995. Intercellular calcium waves mediated by diffusion of inositol triphosphate: a tw0-dimensional model. Am. J. Physiol. 268:C1537-C1545.

Sneyd, J., M. Wilkins, A. Stahonja and M. Sanderson. 1998. Calcium waves and oscillations driven by an intercellular gradient of inositol (1,4,5)-triphosphate. Biophys. Chem. 72:101-109.

Somogyi, R. and W. Stucki. 1991. Hormone-induced calcium oscillations in liver cells can be explained by a simple one-pool model. J. Biol. Chem. 266:11068-11077. 
Stundzia, A. B. and C. J. Lumsden. 1996. Stochastic simulation of coupled reaction-diffusion processes. J. Comp. Phys. 127:196-207.

Thomas, A. P., G. S. J. Bird, G. Hajnoczky, L. D. Robb-Gaspers and J. W. Putney. 1996. Spatial and temporal aspects of cellular calcium signaling. FASEB J. 10:1505-1517.

Thomas, A. P., D. C. Renard-Rooney, G. Hajnoczky, L. D. Robb-Gaspers, C. Lin and T. A. Rooney. 1995. Subcellular organization of calcium signaling in hepatocytes and intact liver. Ciba Foundation Symposia 188:18-35.

Thomas, A. P., D. C. Renard and T. Rooney. 1991. Spatial and temporal organization of calcium signaling in hepatocytes. Cell Calcium 12:111-126.

Tordjmann, Th., B. Berthon, E Jacquemin, C. Clair, N. Stelly, G. Guillon, M. Claret and L. Combettes. 1998. Receptororiented intercellular waves evoked by vasopressin in rat hepatocytes. EMBO J. 17:4695-4703.

Tordjmann, Th., B. Berthon, M. Claret and L. Combettes. 1997. Coordinated intercellular calcium waves induced by noradrenaline in rat hepatocytes: dual control by gap junction permeability and agonist. EMBO J. 16:5398-5407.

Yule, D. I., E. Stuenkel and J. A. Williams. 1996. Intercellular calcium waves in rat pancreatic acini: mechanism of transmission. Am. J. Physiol. 40:C1285-C1294. 
TABLE I. Typical simulation constants for model with intercellular diffusion of $\mathrm{Ca}^{2+}$.

\begin{tabular}{|c|c|}
\hline Parameter & Value \\
\hline$P$ & $2.0 \mu M$ \\
\hline$\nu_{0}$ & $0.2 \mu M s^{-1}$ \\
\hline$\nu_{c}$ & $4.0 \mu M s^{-1}$ \\
\hline$K_{0}$ & $4.0 \mu M$ \\
\hline$\nu_{4}$ & $3.6 \mu M s^{-1}$ \\
\hline$K_{4}$ & $0.12 \mu M$ \\
\hline$\nu_{3}$ & $9.0 \mu M s^{-1}$ \\
\hline$K_{3}$ & $0.12 \mu M$ \\
\hline$k_{1}$ & $40.0 \mathrm{~s}^{-1}$ \\
\hline$d_{1}$ & $0.3 \mu M$ \\
\hline$d_{2}$ & $0.4 \mu M$ \\
\hline$d_{3}$ & $0.2 \mu M$ \\
\hline$d_{p}$ & $0.2 \mu M$ \\
\hline$d_{a}$ & $0.4 \mu M$ \\
\hline$k_{2}$ & $0.02 s^{-1}$ \\
\hline$\rho$ & $0.02 \mu m^{-1}$ \\
\hline$\alpha$ & 2.0 \\
\hline$\beta$ & 0.1 \\
\hline
\end{tabular}


TABLE II. Stochastic formulation of model with intercellular diffusion of $\mathrm{Ca}^{2+}$

\begin{tabular}{cc}
\hline \hline Reaction constant & Stochastic process \\
\hline$a_{j}^{(1)}$ & $X_{j} \rightarrow X_{j}+1$ \\
$a_{j}^{(2)}$ & $X_{j} \rightarrow X_{j}-1$ \\
$a_{j}^{(3)}$ & $Z_{j} \rightarrow Z_{j}+1$ \\
$a_{j}^{(4)}$ & $Z_{j} \rightarrow Z_{j}-1$ \\
$a_{j}^{(5)}$ & $X_{j} \rightarrow X_{j}+1$ \\
& $X_{j+1} \rightarrow X_{j+1}-1$ \\
$a_{j}^{(6)}$ & $X_{j} \rightarrow X_{j}-1$ \\
& $X_{j+1} \rightarrow X_{j+1}+1$ \\
$a_{j}^{(7)}$ & $Z_{j} \rightarrow Z_{j}+1$ \\
& $Z_{j+1}-1$ \\
$a_{j}^{(8)}$ & $Z_{j+1} \rightarrow Z_{j}$ \\
& $Z_{j} \rightarrow Z_{j}-1$ \\
\hline \hline
\end{tabular}


TABLE III. Simulation constants for model with intercellular diffusion of $\mathrm{IP}_{3}$

\begin{tabular}{|c|c|}
\hline Parameter & Value \\
\hline$k_{+}$ & $25.0 s^{-1} \mu M^{-4}$ \\
\hline$k_{-}$ & $2.5 \times 10^{-3} s^{-1}$ \\
\hline$K_{a c t}$ & $0.34 \mu M$ \\
\hline$k_{1}$ & $42.0 s^{-1} \mu M^{-1}$ \\
\hline$b$ & $10^{-4}$ \\
\hline$V_{M P}$ & $8.0 \mu \mathrm{M} / \mathrm{s}$ \\
\hline$K_{p}$ & $0.4 \mu M$ \\
\hline$\alpha$ & 0.1 \\
\hline$C a_{t o t}$ & $60.0 \mu M$ \\
\hline$K_{I P}$ & $1 \mu M$ \\
\hline$V_{K}$ & $7.5 \times 10^{-3} \mu \mathrm{M} / \mathrm{s}$ \\
\hline$V_{P H}$ & $7.5 \times 10^{-2} \mu \mathrm{M} / \mathrm{s}$ \\
\hline$K_{K}$ & $1 \mu M$ \\
\hline$K_{P H}$ & $10 \mu M$ \\
\hline$K_{d}$ & $0.5 \mu M$ \\
\hline$D_{C a}$ & $30 \mu m^{2} / \mathrm{s}$ \\
\hline$D_{I P}$ & $210 \mu m^{2} / s$ \\
\hline$F_{I P}$ & $0.35 \mu \mathrm{m} / \mathrm{s}$ \\
\hline
\end{tabular}



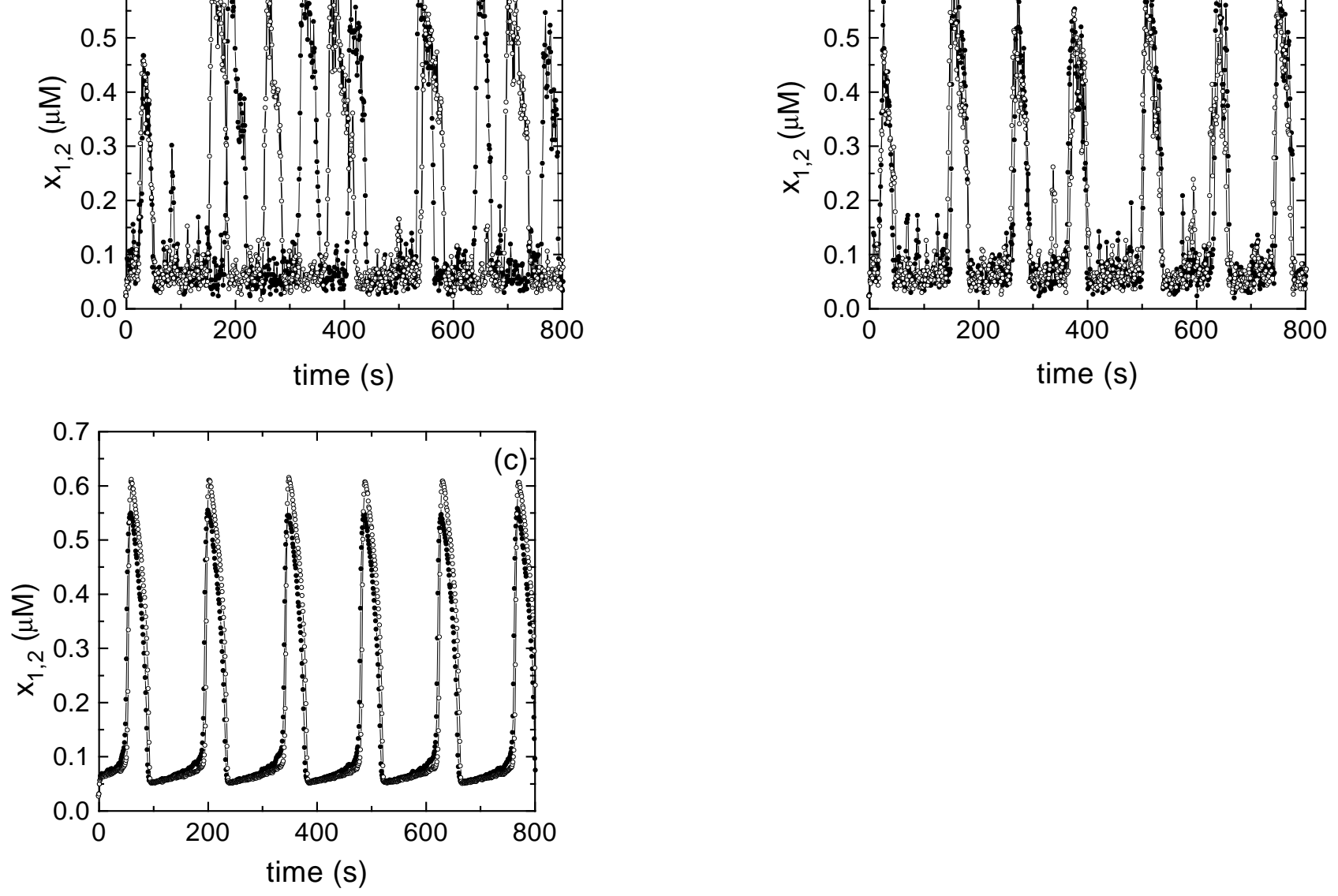

Figure 3.

Calcium oscillations for a doublet of cells, $N=2$, for different values of the permeability constant $\gamma$ : (a) $\gamma=0 s^{-1}$, (b) $\gamma=0.07 \mathrm{~s}^{-1}$. Values of parameters are as in table I with $\rho_{1}=\rho_{2}=0.02, \beta_{1}=0.15, \beta_{2}=0.2, P_{1}=P_{2}=2.0$ and the cell volume is $\Omega=300 \mu \mathrm{m}^{3}$. Same symbols meaning that in figure 2 . In figure (c) we show the calcium oscillation in the deterministic limit obtained for $\Omega=10^{5}$ and $\gamma=0.07 \mathrm{~s}^{-1}$. 

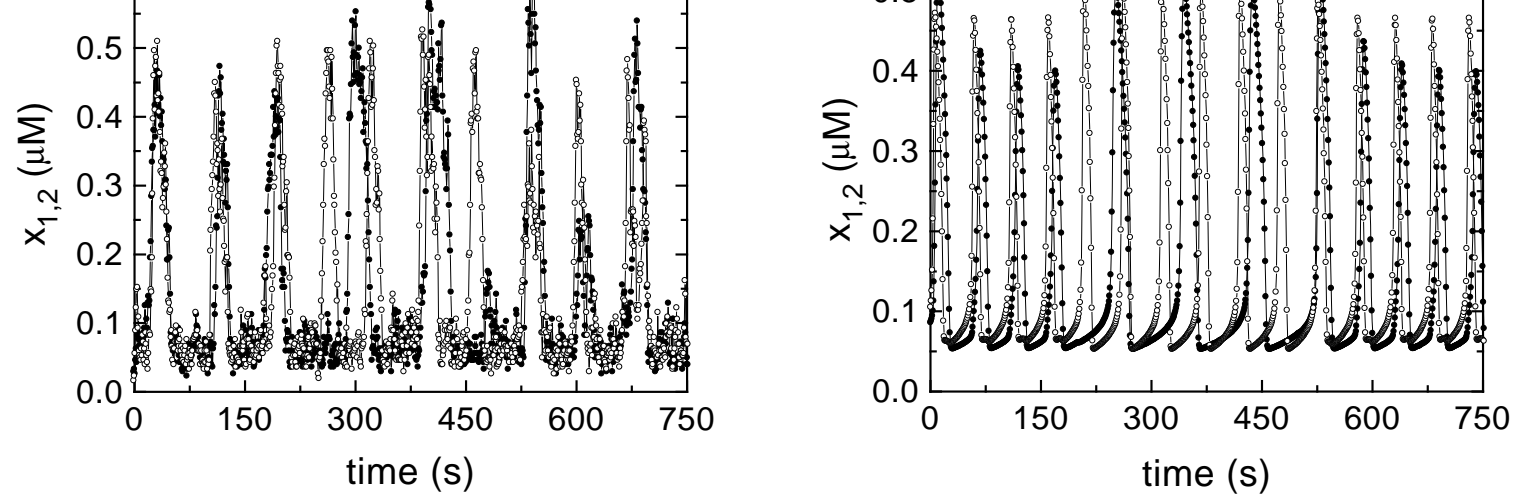

Figure 4.

Effect of the temporal blocking of gap junction between two connected cells in the stochastic version of Höfer's model. We take $\gamma=0 \mathrm{~s}^{-1}$ for time $=200-500 \mathrm{~s}$ and $\gamma=0.06 \mathrm{~s}^{-1}$ otherwise. Figure (a) takes $\Omega=300 \mu \mathrm{m}^{3}, \rho_{1}=0.015, \rho_{2}=0.02$, $\beta_{1}=\beta_{2}=0.1$, while in figure (b) we consider the deterministic limit with $\Omega=10^{5}$ (this is the analog of Fig 6(a) from (Höfer et al., 1999)). Other parameters and symbol meanings as in figure 3. 

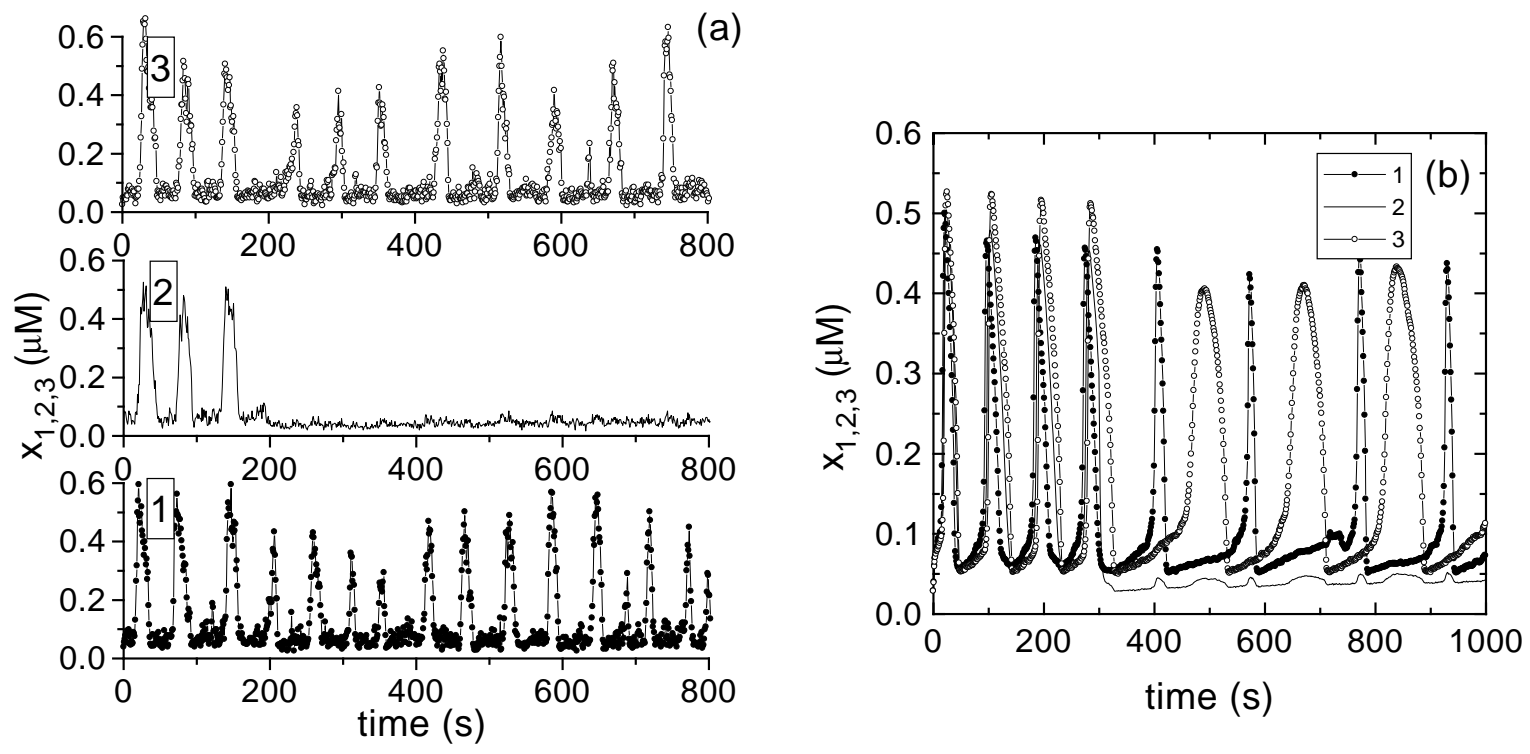

Figure 5 .

Simulation of heparin treatment for middle cell in the stochastic version of Höfer's model for a cell triplet, $N=3$. The treatment starts at time $=200 \mathrm{~s}$. We have used the following parameters: $P_{2}=P_{2}=P_{3}=2 \mu \mathrm{M}, \rho_{1}=0.025, \rho_{2}=0.018, \rho_{3}=0.02$, $\beta_{1}=\beta_{2}=\beta_{3}=0.1, \gamma=0.07 \mathrm{~s}^{-1}$. We plot the time series of the variables $x_{1}, x_{2}$ and $x_{3}$ in the cases (a) $\Omega=300 \mu m^{3}$ where stochastic effects are important, and (b) in the deterministic limit with $\Omega=10^{5}$, analog of Fig 6(b) from (Höfer et al., 1999), where treatment starts at time $=300 \mathrm{~s}$. 


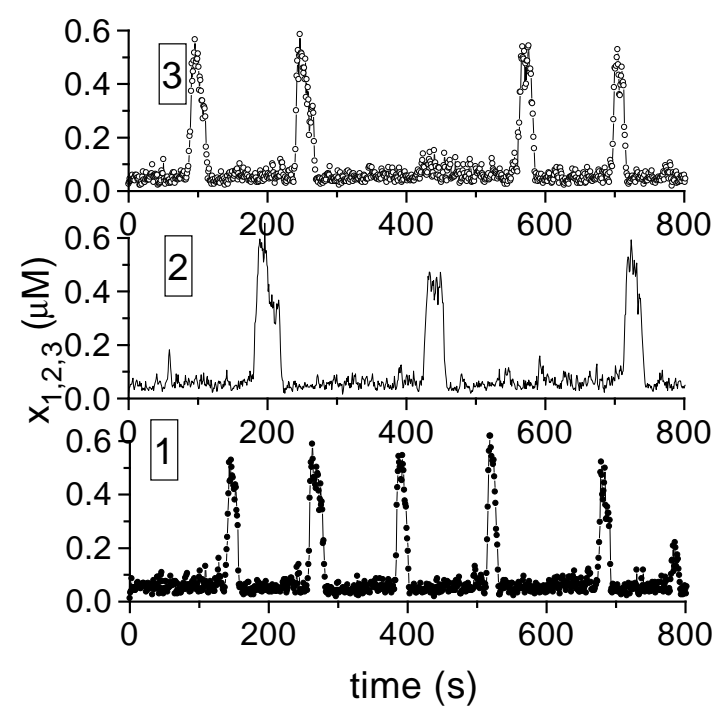

Figure 6.

Calcium signaling for the stochastic version of Höfer's model in the case of three cells, $N=3$, for a cell volume $\Omega=300 \mu m^{3}$ in the case of blocked gap junctions: $\gamma=0 \mathrm{~s}^{-1}$. Parameters are as in table I with $P_{1}=P_{2}=P_{3}=1 \mu \mathrm{M}, \beta_{1}=\beta_{2}=\beta_{3}=0.1$. We have considered that the three cells have different structural parameters: $\rho_{1}=0.025, \rho_{2}=0.015, \rho_{3}=0.02$. 

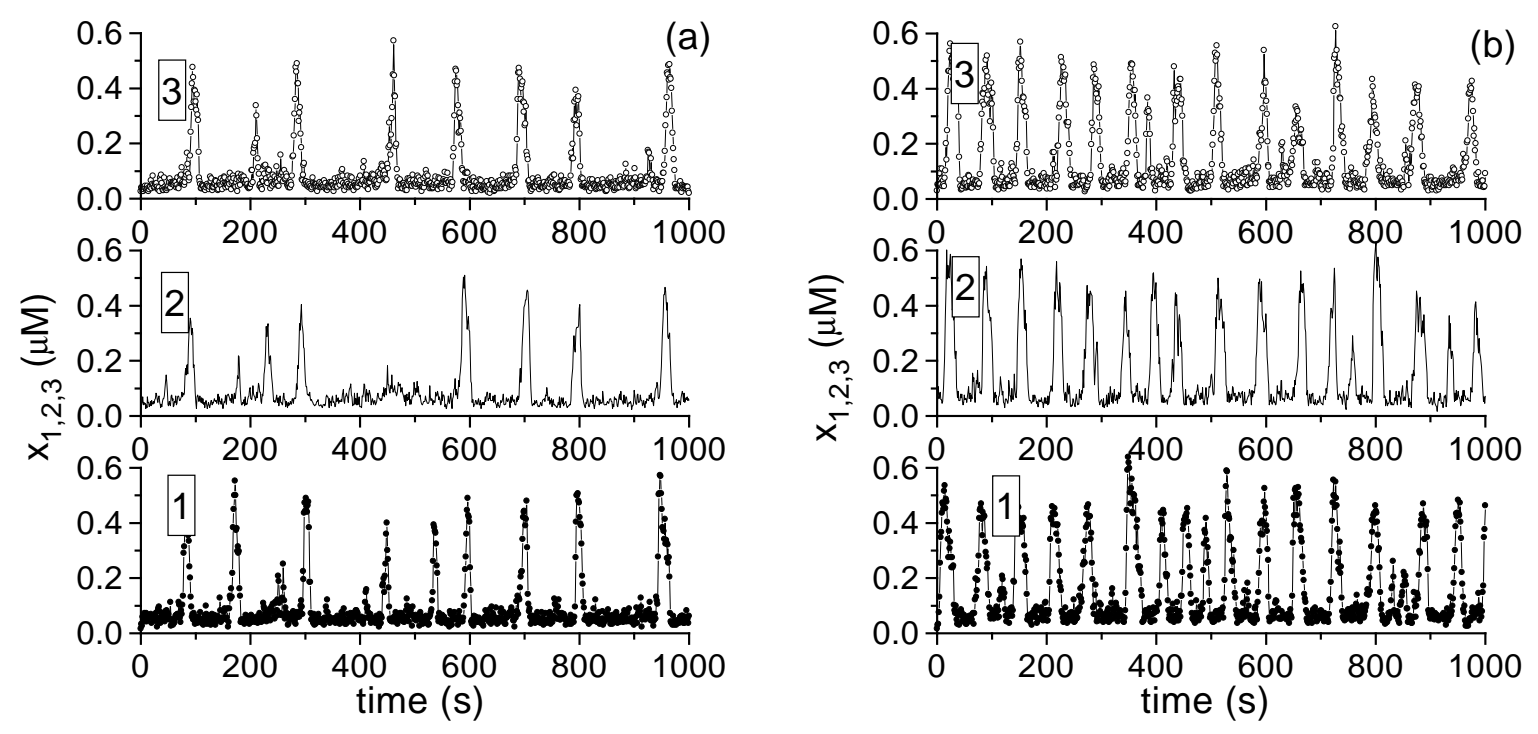

Figure 7.

Results for the low stimulus calcium oscillations in the stochastic version of Höfer's model for three connected cells and $\Omega=300 \mu \mathrm{m}^{3}$. (a) $\rho_{1}=0.025, \rho_{2}=0.015, \rho_{3}=0.02, P_{1}=P_{2}=P_{3}=1 \mu \mathrm{M}, \gamma=0.07^{-1}$, (b) $\rho_{1}=\rho_{2}=\rho_{3}=0.027$, $P_{1}=1.2 \mu \mathrm{M}, P_{2}=1.1 \mu \mathrm{M}, P_{3}=1.0 \mu \mathrm{M}, \gamma=0.03^{-1}$. 


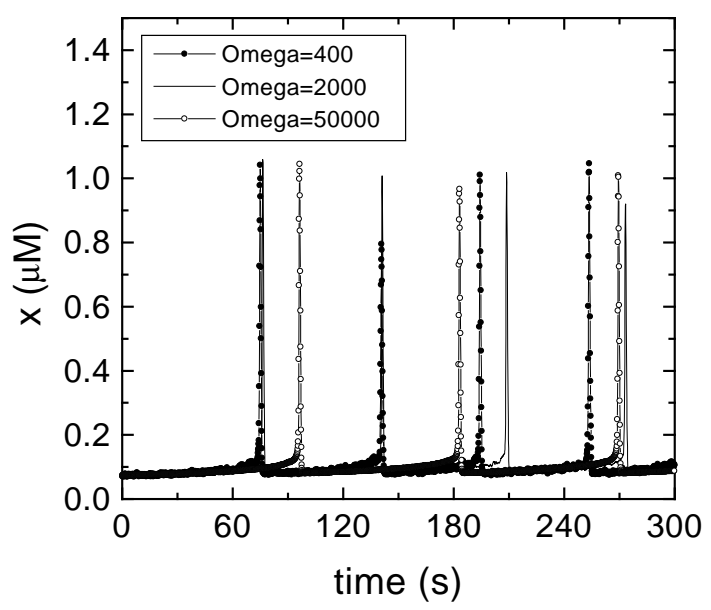

Figure 8 .

Results of calcium oscillations in one cell for the stochastic version of Dupont et al. model for values of $\Omega=400,2000,50000$. Notice that, as expected, fluctuations decrease with increasing $\Omega$ and that the deterministic limit is already well reproduced by $\Omega=50000$. Initial conditions are resting states corresponding to $\mathrm{V}_{P L C}=6.5 \times 10^{-4} \mu \mathrm{M} / \mathrm{s}$. The rest of parameters are in table III. 


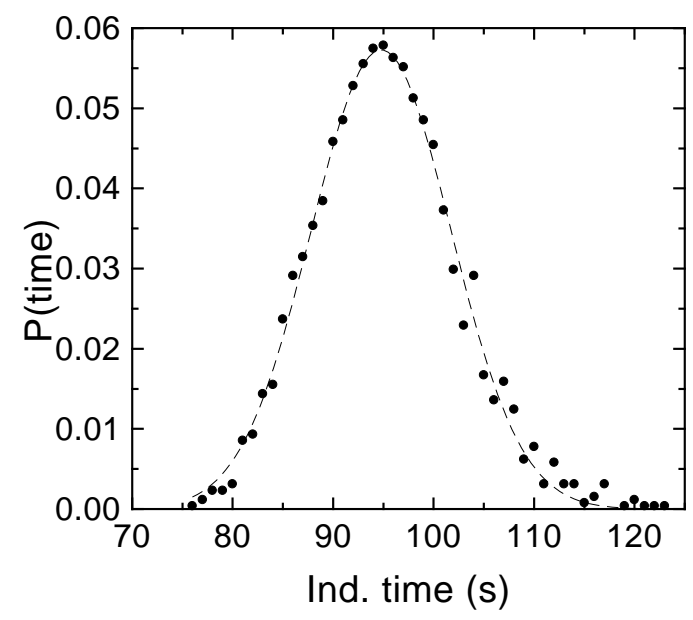

Figure 9.

Distribution of induction times coming from the stochastic version of Dupont et al. model for a single cell with $\Omega=400$, $V^{P L C}=2 \times 10^{-3} \mu \mathrm{M} / \mathrm{s}$. Other parameters as in table III. 

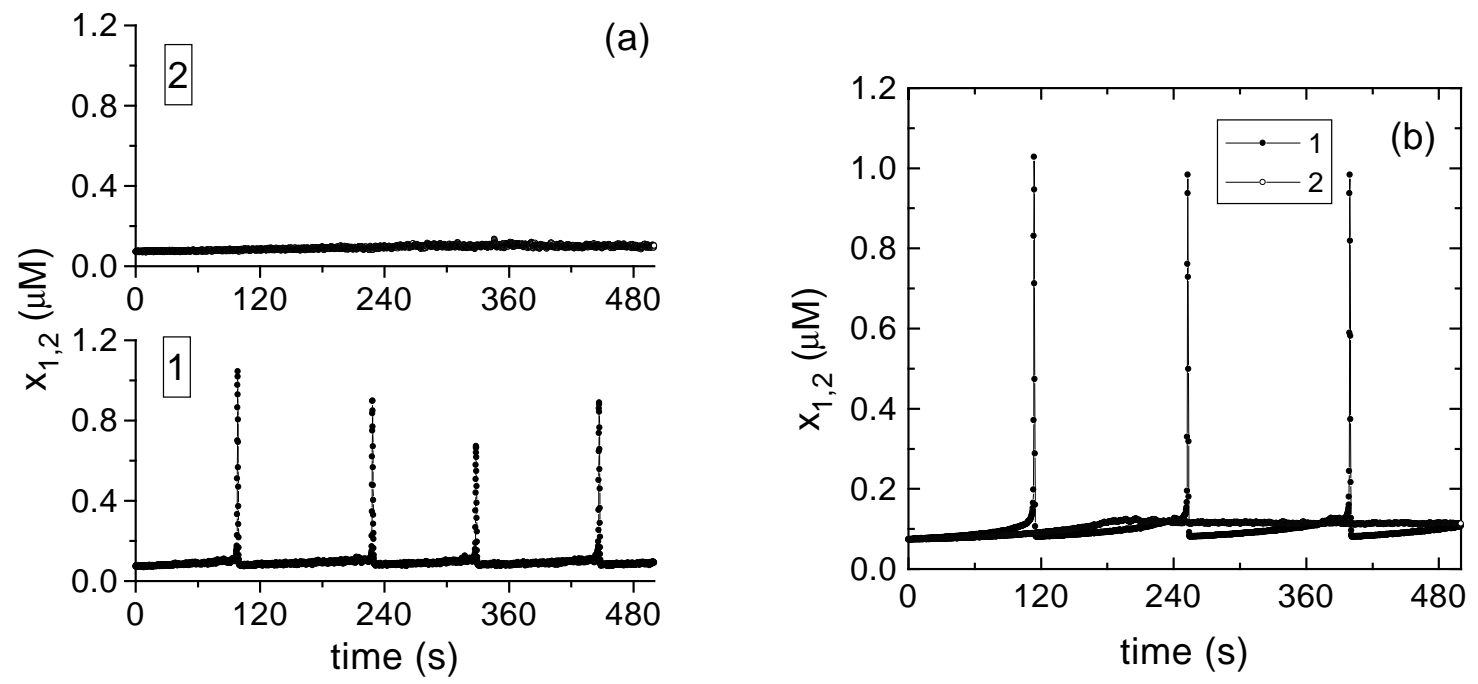

Figure 10.

Calcium oscillations in two connected cells as obtained from the stochastic version of Dupont et al. model. Only the first cell is stimulated. We use this model experiment as a method of adjusting the membrane permeability $F_{I P}$ since it is known that stimulation of just one cell does not produce response in the second cell. (a) Genuine stochastic case with $\Omega=400$, $F_{I P}=0.35, V_{1}^{P L C}=2.5 \times 10^{-3} \mu \mathrm{M} / \mathrm{s}, V_{2}^{P L C}=6.5 \times 10^{-4} \mu \mathrm{M} / \mathrm{s}$, other parameters as in table III. (b) Deterministic limit obtained by taking the large value $\Omega=50000$. Following the parameters used in (Dupont et al., 2000) (Fig. 3c) we set $\mathrm{F}_{I P}=0.88$, $V_{1}^{P L C}=2.77 \times 10^{-3} \mu \mathrm{M} / \mathrm{s}, V_{2}^{P L C}=6.5 \times 10^{-4} \mu \mathrm{M} / \mathrm{s}$ and the rest of the parameters as in table III. 

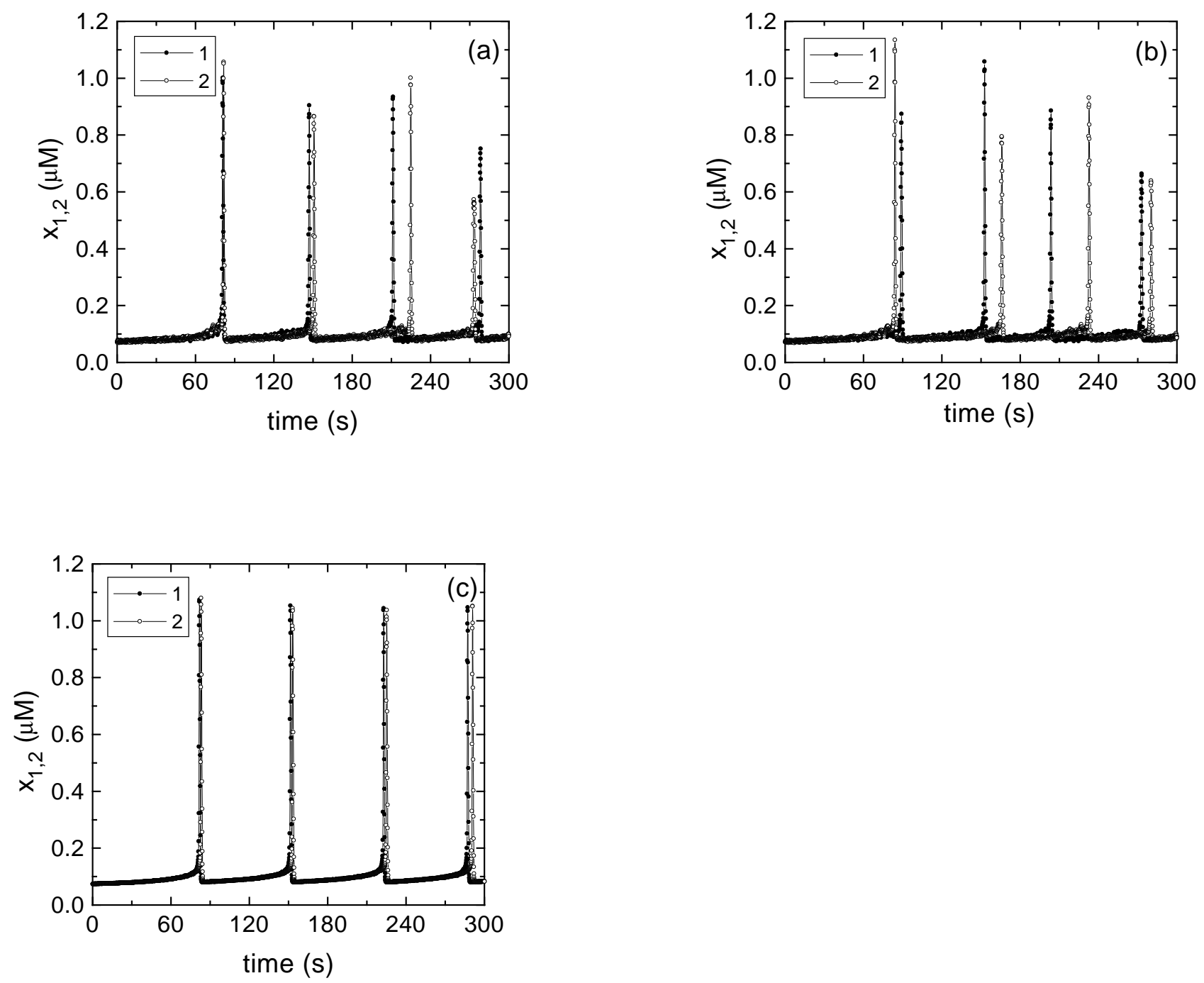

Figure 11.

Calcium oscillations in two connected cells. Both cells are stimulated. Figures (a) and (b) correspond to the genuine stochastic case while figure (c) is the deterministic limit. Parameters as in table III and (a) $\Omega=400, \mathrm{~F}_{I P}=0.35, V_{1}^{P L C}=$ $2.2 \times 10^{-3} \mu M / s, V_{2}^{P L C}=2.1 \times 10^{-3} \mu M / s ;$ (b) $\Omega=400, F_{I P}=0.0, V_{1}^{P L C}=2.2 \times 10^{-3} \mu M / s, V_{2}^{P L C}=2.1 \times 10^{-3} \mu M / s$; (c) $\Omega=50000$ (deterministic limit) and $\mathrm{F}_{I P}=0.88, V_{1}^{P L C}=2.205 \times 10^{-3} \mu \mathrm{M} / \mathrm{s}, V_{2}^{P L C}=2.1 \times 10^{-3} \mu \mathrm{M} / \mathrm{s}$, to match those used in (Dupont et al., 2000) (figure 3d). 

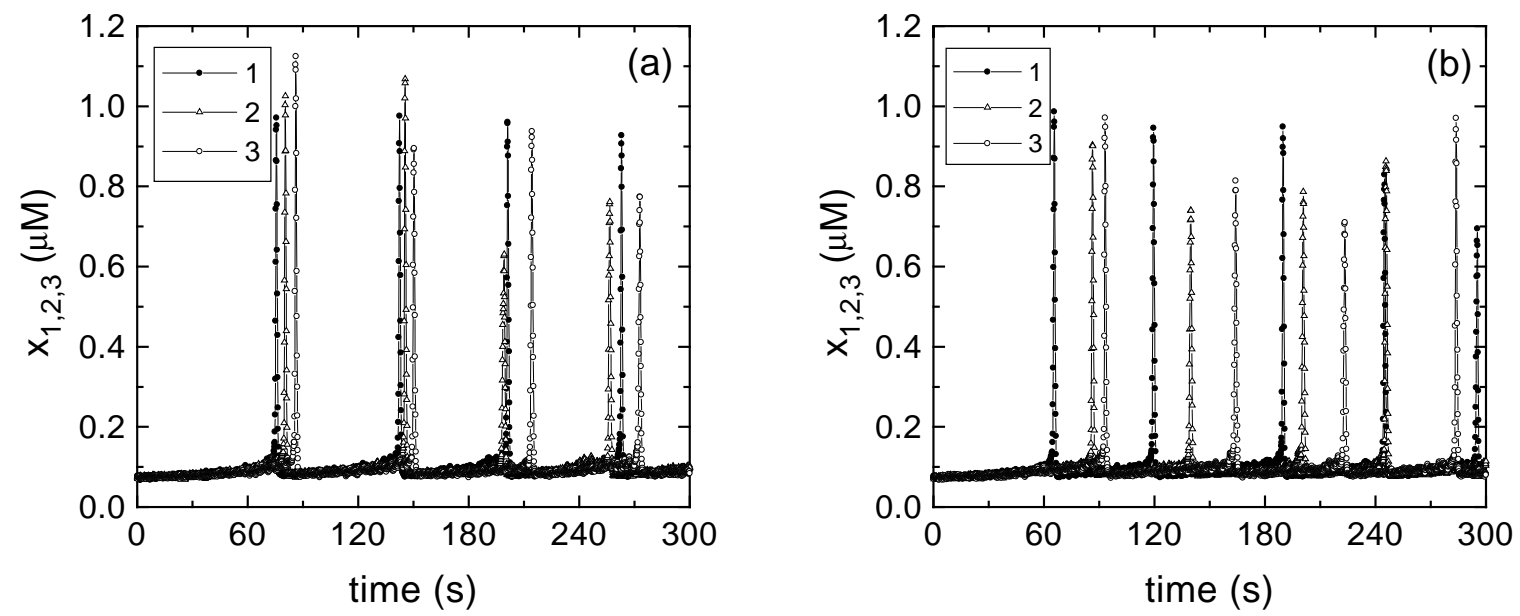

Figure 12.

Calcium oscillations in three connected cells using the stochastic version of Dupont et al. model. Parameters as in table III and $\Omega=400, V_{1}^{P L C}=2.3 \times 10^{-3} \mu M / s, V_{2}^{P L C}=2.2 \times 10^{-3} \mu M / s, V_{3}^{P L C}=2.1 \times 10^{-3} \mu M / s$. (a) $\mathrm{F}_{I P}=0.35$, (b) $\mathrm{F}_{I P}=0.0$. 

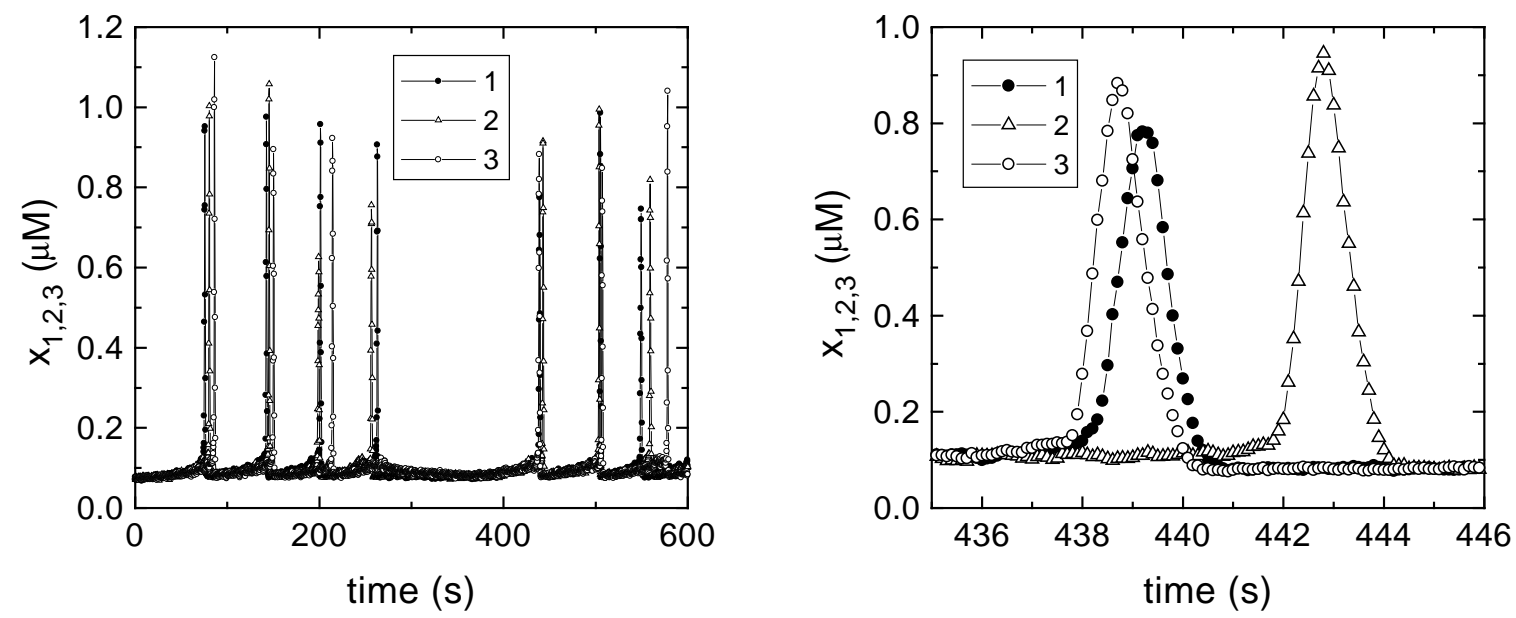

Figure 13.

(a) Numerical experiment using the stochastic version of Dupont et al. model for three connected cells with agonist removal right before third cell spikes at $t=258 \mathrm{~s}$. After restoration of agonist at $t=358 \mathrm{~s}$ third cell spikes first. Parameters the same as for Fig. 12(a). (b) Details of Fig. 13(a) near $t=358 \mathrm{~s}$. 